\title{
Systemic Weber-Christian disease with reversible bilateral ureteric obstruction
}

\author{
L.A. Brawn, L.E. Ramsay, J.R. Shortland and J.L. Williams
}

University Departments of Therapeutics, Urology and Pathology, Royal Hallamshire Hospital, Glossop Road, Sheffield S10 2JF, UK.

\begin{abstract}
Summary: It has been proposed that idiopathic retroperitoneal fibrosis may be a consequence of 'healed' retroperitoneal lesions of systemic Weber-Christian disease. However ureteric obstruction which is the hallmark of idiopathic retroperitoneal fibrosis, has not been described in systemic Weber-Christian disease. We report a patient with systemic Weber-Christian disease who, during a relapse, developed bilateral ureteric obstruction which resolved when the Weber-Christian disease remitted. The radiological appearances were consistent with a diagnosis of idiopathic retroperitoneal fibrosis, but the clinical course was slightly atypical in that the ureteric obstruction resolved completely and rapidly. Ureteric obstruction can complicate systemic Weber-Christian disease and this observation gives support to the hypothesis that idiopathic retroperitoneal fibrosis is related to systemic Weber-Christian disease.
\end{abstract}

\section{Introduction}

Chronic panniculitis (necrosis of subcutaneous fat) may be associated with a definable underlying disorder, such as systemic lupus erythematosus, pancreatic disease, alpha ${ }_{1}$-antitrypsin deficiency, neoplasia or trauma. ${ }^{1}$ The term Weber-Christian disease defines a chronic disorder with no identifiable cause characterized by relapsing nodular panniculitis and fever. ${ }^{1}$ Two forms of Weber-Christian disease are recognized. ${ }^{2,3}$ In Weber-Christian panniculitis the lesions are confined to subcutaneous adipose tissue, and in systemic Weber-Christian disease lesions are found in visceral adipose tissue as well as subcutaneous fat. ${ }^{3}$ In the few cases of systemic WeberChristian disease which have been described fully, retroperitoneal lesions have invariably been present, with less constant involvement of the mediastinum, pericardium, myocardium and bladder neck. ${ }^{3}$

Milner and Mitchinson ${ }^{3,4}$ have drawn attention to similarities between the visceral abnormalities of systemic Weber-Christian disease and certain chronic fibrotic disorders such as systemic idiopathic fibrosis, retractile mesenteritis, and idiopathic retroperitoneal fibrosis (IRF). Mitchinson ${ }^{4}$ suggested that systemic idiopathic fibrosis might be a form of Weber-Christian disease in which subcutaneous panniculitis was either absent or overlooked, and that IRF might be one manifestation of the more widespread disorder

Correspondence: L.E. Ramsay, M.B., F.R.C.P. University Department of Therapeutics, Royal Hallamshire Hospital, Glossop Road, Sheffield S10 2JF, UK.

Accepted: 19 October 1988 systemic idiopathic fibrosis. Milner and Mitchinson ${ }^{3 \mathrm{C}}$ further pointed out that 'healed' retroperitonealo Weber-Christian disease might resemble IRF. The $\overrightarrow{0}$ complex relations between IRF, systemic idiopat $c_{0}^{\circ}$ fibrosis, and systemic Weber-Christian disease p posed by these workers ${ }^{3,4}$ and echoed by others ${ }^{5,6}$ afeo shown in Figure 1. This hypothesis is important because it could cast some light upon the aetiology and evolution of IRF, the most common of the conditionso mentioned. However, the hypothesis has at least one major weakness. Although retroperitoneal lesionsoํㅡㄹ have invariably been present at autopsy in systemic Weber-Christian disease, ${ }^{3}$ ureteric obstruction, which is so typical of IRF, has not been reported in this. condition.

We describe a patient who developed bilatera: ureteric obstruction as a complication of systemic Weber-Christian disease, and discuss the relevance of this observation to the hypothesis that IRF may be as consequence of 'healed' retroperitoneal Weber-o Christian disease. In addition, the lesions in this? patient showed vasculitis and deposition of immuno $\frac{7}{0}$ globulin and complement, features which support an immunological basis for systemic Weber-Christiano disease.

\section{Case report}

A 48 year old man was admitted in April 1985 with fever, abdominal pain, weight loss, and a sub $=$ cutaneous swelling of the right loin.

(C) The Fellowship of Postgraduate Medicine, 1989? 


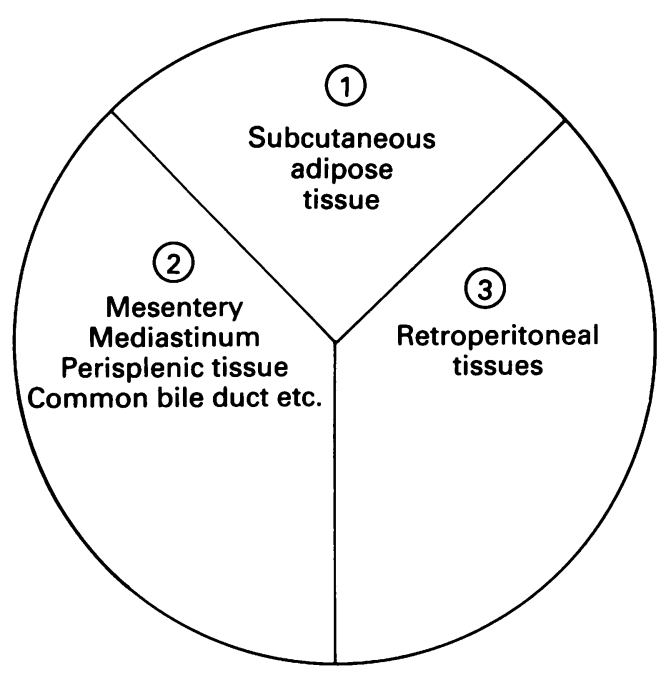

(1) Alone: Weber-Christian panniculitis

(1) + (2) + (3): Systemic Weber-Christian disease

(2) + (3): Systemic idiopathic fibrosis

(3) Alone: Idiopathic retroperitoneal fibrosis

Figure 1 Interrelations between IRF, Weber-Christian disease and systemic idiopathic fibrosis as proposed by Mitchinson et al. ${ }^{4}$

He had suffered headaches, diagnosed as migraine, since the age of 23 years. From the age of 34 he had bouts of fever, as high as $39^{\circ} \mathrm{C}$, lasting $2-12$ hours and occurring approximately once weekly. When aged 37 he developed progressive visual failure which resulted in complete blindness two years later. Fundoscopy was said to show 'poor vasculature' and macular degeneration, and his ESR was persistently elevated. Treatment with prednisolone for 8 months did not lower the ESR or halt the progression of his condition, which was diagnosed as a 'heredofamilial retinopathy'. At the age of 43 , he had abdominal pain and pyrexia which persisted for 4 months, and was investigated extensively elsewhere. The positive findings were a mild anaemia of chronic disorder, elevated ESR between $60-120 \mathrm{~mm} / \mathrm{h}$, and slightly elevated serum transaminases. Despite numerous microbiological, immunological and radiological investigations, and biopsies of bone marrow and liver, no diagnosis was reached and his symptoms eventually resolved spontaneously. One year later, when aged 44 , he first noted transient palpable subcutaneous swellings on his legs, back or abdomen. These nodules would develop suddenly after a few days of fever, were not tender or discoloured, and resolved without scarring. Their size varied from that of a pea to that of an orange. At the age of 45 , three years before admission, he had a brief episode of loin pain. An intravenous pyelogram was normal.

On admission he gave a 3-month history of dull central abdominal pain radiating to the back, anorexia, vomiting, weight loss of $12 \mathrm{~kg}$, persistent pyrexia and an increase in the frequency of his subcutaneous swellings. His family doctor had found that his ESR was elevated, and had treated him with prednisolone and naproxen without benefit. Examination showed blindness, bilateral optic atropy and pyrexia as high as $39^{\circ} \mathrm{C}$. He had a palpable non-tender subcutaneous swelling measuring $9 \times 6 \mathrm{~cm}$ on his right loin, and a $0.5 \times 0.5 \mathrm{~cm}$ nodule on his left leg. Initial investigation showed haemoglobin $10.1 \mathrm{~g} / \mathrm{dl}$; ESR $132 \mathrm{~mm} / \mathrm{h}$; serum gamma glutamyl transaminase $43 \mathrm{IU} / \mathrm{l}$ (normal < 30) and aminoaspartate transferase $44 \mathrm{IU} / 1$ (<26). Serum bilirubin, ALT, alkaline phosphatase, urea, creatinine, electrolytes and glucose, urine chemistry and microscopy, and cultures of urine, blood and faeces were all normal.

The subcutaneous nodule on his right loin was biopsied. The histological appearances were typical of Weber-Christian disease and are described in detail below.

Intravenous pyelography showed bilateral dilatation of the upper urinary tract, more marked on the left, with some medial displacement of the ureters (Figure 2a). Retrograde pyelography confirmed dilatation of both upper ureters, with narrowing of the lower $6 \mathrm{~cm}$, and the catheter traversed the narrowed part of each ureter without difficulty. There was no filling defect in the bladder. These findings were consistent with a diagnosis of idiopathic retroperitoneal fibrosis.

An abdominal computed tomographic (CT) scan showed bilateral hydronephrosis and the fat between the bladder and rectum had a higher attenuation than normal. There was no evidence of a retroperitoneal tumour or mass. Barium enema and barium meal and follow-through showed no abnormality. He had never taken methysergide or any other drug suspected of causing retroperitoneal fibrosis.

Additional investigations showed normal serum amylase, lipids, alpha $a_{1}$-antitrypsin and antinuclear factor. The pancreas showed no abnormality on ultrasound and CT scan examination. Assay of serum complement showed persistent reduction of the $\mathrm{C}_{4}$ component $(<0.1 \mathrm{~g} / \mathrm{l}$, normal $0.2-0.65 \mathrm{~g} / \mathrm{l})$.

During investigation he remained pyrexial and ill, the ESR rose to $146 \mathrm{~mm} / \mathrm{h}$, and his renal function declined slightly (serum creatinine $122 \mu \mathrm{mol} / 1$, normal $<120 \mu \mathrm{mol} / \mathrm{l}$ ). A provisional diagnosis of systemic Weber-Christian disease with retroperitoneal involvement causing bilateral ureteric obstruction was reached. He was treated with indomethacin, $50 \mathrm{mg}$ thrice daily, with a rapid subjective response. Three 

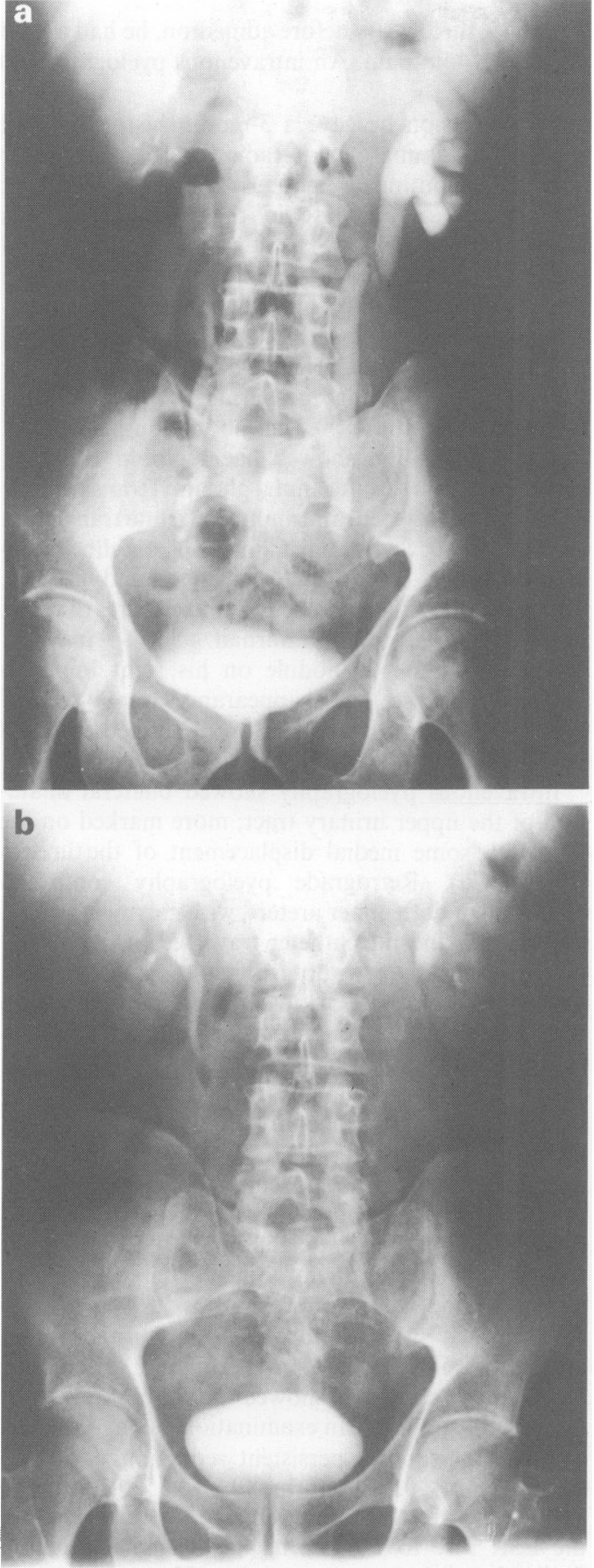

Figure 2 Intravenous pyelography (a) during relapse of Weber-Christian disease; (b) 3 months later during remission. Note the bilateral ureteric obstruction in (a) with the narrowing confined to the lower $3 \mathrm{~cm}$ of both ureters and some medial displacement of the ureters. Complete resolution is seen in (b). months later he felt 'better than he had for years', with no fever, abdominal pain or recurrence of subcutaneous nodules. His weight had increased by $6 \mathrm{~kg}$. The haemoglobin had risen to $13.2 \mathrm{~g} / \mathrm{dl}$, the ESR had fallen to $15 \mathrm{~mm} / \mathrm{h}$, and the serum creatinine and transaminases had returned to normal. A repeat intravenous pyelogram showed complete resolution of the ureteric obstruction (Figure $2 b$ ).

\section{Pathology}

Macroscopically the biopsy consisted of mildly congested yellowish-grey fibrofatty tissue. The histological features were of an established panniculitis with a prominent vasculitic component. Fibrous septae separated severely and minimally involved lobules which were adjacent to each other (Figure 3a). The uneven distribution of the inflammatory changes between lobules was particularly characteristic of Weber-Christian disease.

The least damaged lobules showed microscopic foci of lymphocytes and polymorphonuclear leucocytes infiltrating the walls of small venules and adjacent fat (Figure $3 \mathrm{~b}$ ). In the more affected areas there were clusters of histiocytes, lymphocytes and plasma cells with an occasional giant cell. Cyst-like structures were formed by histiocytes surrounding a central clear vacuole and gave the impression of granulomata. The most severely affected lobules were heavily infiltratee by lymphocytes, plasma cells, foamy macrophages and giant cells of both Touton and foreign body type, interspersed with fat spaces. Abundant eosinophils were present throughout the inflammatory infiltrate and accompanied the mild lymphocytic infiltrate extending into the fibrous septae. Discrete granulomata were also identified adjacent to the septae and within these lobules.

The small veins in the terminal septae had grossly thickened walls which were heavily infiltrated by lymphocytes. Surviving smooth muscle cells were swollen with a clear cytoplasm (Figure 3c). The lumina of these veins although narrow, were infrequently occluded. The small arteries and arterioles also displayed endothelial swelling with thickened walls and segmental loss of definition of the smooth muscle cells. These changes were not confined to the terminal septae as veins and occasionally arteries within larger septae also showed evidence of vasculitis.

Immunopathological studies showed $\mathrm{C}_{3}, \mathrm{C}_{4}$ and IgA in the small veins of minimally involved areas (Figure 3d). However, more severely involved venules showed no focal deposits of immunoglobulin or complement fractions despite grossly infiltrated and thickened walls. The thin line-like interstices between the clear cells were outlined by using antibodies to $\operatorname{IgG}, \operatorname{IgA}, \mathrm{C}_{3}$ and fibrin. 

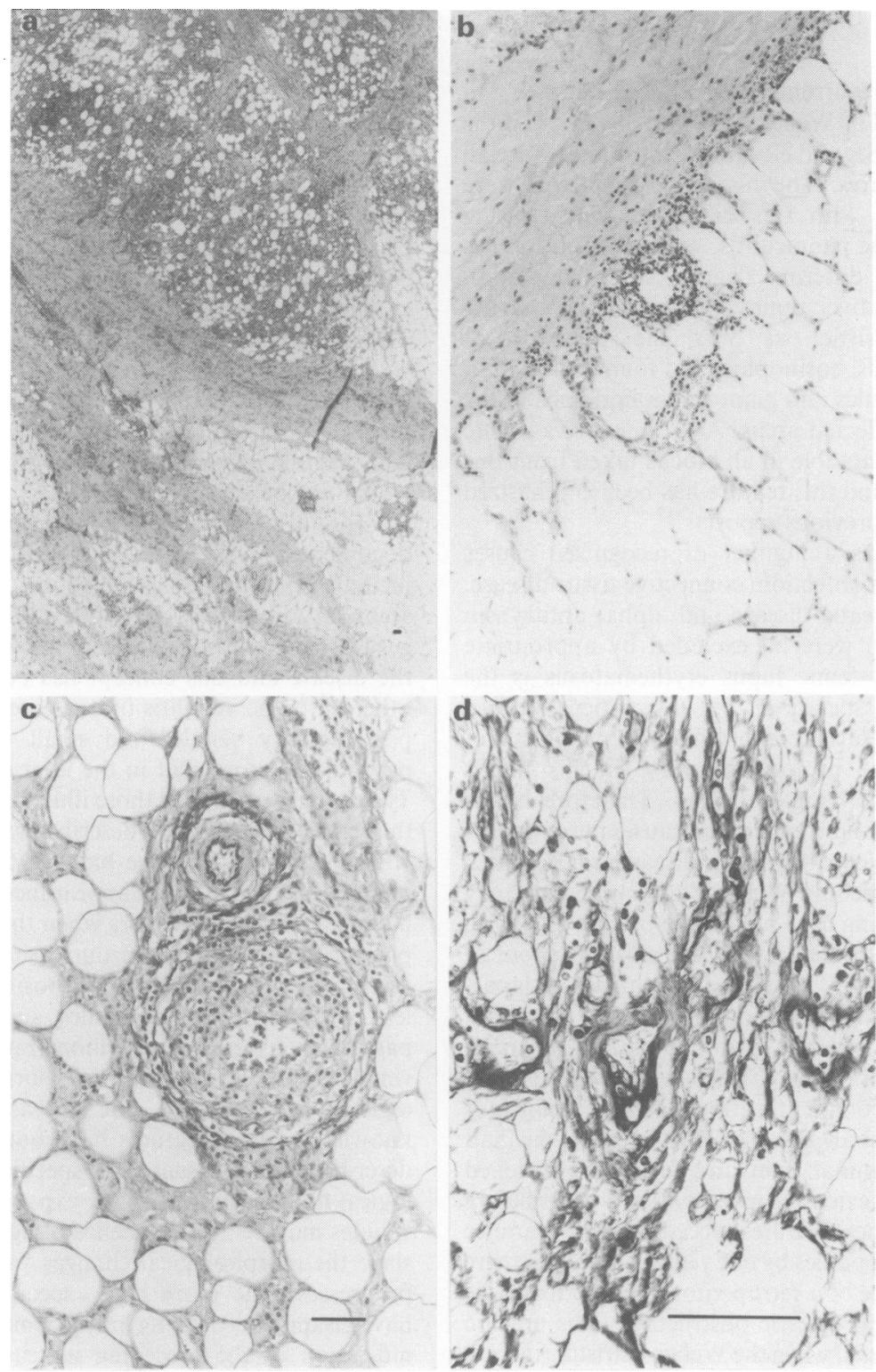

Figure 3 Histology ( $\mathrm{bar}=100 \mu \mathrm{m}$ ). (a) Adjacent lobules of fat separated by a fibrous septum display differing degrees of inflammation. Haematoxylin and eosin. (b) The earliest lesions with inflammatory cells infiltrating the wall of a small venule adjacent to the septum. Haematoxylin and eosin. (c) The wall of this small vein deep in the lobule is heavily infiltrated by inflammatory cells. The adjacent arteriole shows mild endothelial swelling only. Elastic van Gieson. (d) The third component of the complement cascade is seen in the wall of a minimally inflamed lobular venule. Immunoperoxidase $\mathrm{C}_{3}$. 


\section{Discussion}

The history of recurrent subcutaneous nodules and fever was typical of Weber-Christian disease, and the anaemia, high ESR and elevated transaminases are all recognized features. The histological findings were also in keeping with this diagnosis. The striking irregularity of the panniculitis, with adjacent lobules showing widely differing degrees of inflammation separated by relatively uninvolved septae was particularly characteristic, as was the presence of monunuclear cells, eosinophils and foam cells within the involved lobules and giant cell granulomas in the more severely affected areas. A prominent vasculitic component was notable in all blocks taken from this patient's lesion and this feature has been emphasized in a number of previous reports. ${ }^{2,4,7-9}$

Panniculitis has a number of recognized causes including trauma, infection, connective tissue disease, neoplasia, pancreatic disease and alpha ${ }_{1}$ antitrypsin deficiency. These were all excluded by appropriate investigation. Systemic lupus erythematosus is the most common association ${ }^{10}$ but our patient did not have the clinical or laboratory features diagnostic of this disease. The prolonged course of his illness excluded an underlying neoplasm. Therefore in the absence of any of these underlying causes the diagnosis of 'idiopathic' Weber-Christian disease was reached. Visceral involvement in Weber-Christian disease may be suspected during life, ${ }^{1}$ but can be proven only with great difficulty. ${ }^{3}$ In the present case absolute proof of visceral involvement would have required a laparotomy, and this was not thought justified. However, there can be little doubt that this patient did have visceral lesions of Weber-Christian disease. Abdominal pain was a prominent feature at times of relapse of the Weber-Christian disease. Furthermore, he had prolonged abdominal pain and fever which defied diagnosis despite extensive investigation 5 years before admission, and these features preceded the appearance of subcutaneous nodules by one year. In addition, there was clear evidence of a retroperitoneal abnormality as shown by bilateral ureteric obstruction. This ureteric obstruction occurred when the Weber-Christian disease was in severe relapse and resolved completely when the disease remitted. No other cause of ureteric obstruction was found. We conclude therefore that the patient did have systemic Weber-Christian disease.

This case has particular relevance to the relationship proposed between idiopathic retroperitoneal fibrosis (IRF), systemic idiopathic fibrosis and systemic Weber-Christian disease outlined in the introduction and shown in Figure 1. The invariable presence of retroperitoneal involvement at autopsy in systemic Weber-Christian disease ${ }^{3}$ lends credence to the suggestion that IRF could be a consequence of 'healed' retroperitoneal Weber-Christian lesions. ${ }^{3}$ However, ureteric obstruction, which is the hallmark of IRF, h\$ not to our knowledge been described as a complication of systemic Weber-Christian disease. One case ${ }^{11}$ retroperitoneal fibrosis with fibrosis of the surg cutaneous adipose tissue has been described, but there was no ureteric obstruction and the clinical and pathological features were not typical of Weber? Christian disease. The fact that ureteric obstruction complicated systemic Weber-Christian disease in ow case lends support to the hypothesis of Mitchinson The radiological appearances were entirely consiste with a diagnosis of IRF. Clinically the picture w slightly atypical in that the ureteric obstruction resolved completely with conservative management and without corticosteroid therapy. This outcome would not be anticipated in IRF.

Vasculitis has been reported in about $50 \%$ क् documented case reports of Weber-Christian pas. niculitis $^{8}$ but it has usually been interpreted as $\&$ secondary phenomenon. McDonald and Feiwel sugegested that there might be an immunological basis fơ the disease and this concept has been supported by others. ${ }^{12,13}$ The venulitis in our case was observed post-capillary venules and small venules and was particularly prominent in the least damaged lobules. The changes resembled those illustrated by Unger ${ }^{7}$ but the very early changes he described were not identifie It is noteworthy that the biopsy was taken appegximately 24 hours after the appearance of the nodule his case in contrast to ours when the lesion had bein present for some days. In our case the venulitis associated with complement deposition $\left(C_{3} C_{4}\right)$ in the less severely involved lobules suggesting classica्द pathway activation. The demonstration of $\operatorname{IgA}$ in th venular walls gives further support to an immuno logical aetiology for the disease and, to oo $\overrightarrow{\vec{A}}$ knowledge, these features have not previously beeñ described. The absence of specific immunopathot logical findings in the grossly expanded and inflamed venules may be a consequence of ageing of the lesion since the morphological changes vary as the disease progresses. The more labile specific reactants may have disappeared leaving only plasmatic markers as an indication of the preceding increased permeability This uneven pattern of involvement might be explained by the relapsing nature of the disease wit crops of vasculitic lesions appearing during a relapse

Recently, a vascular aetiology has been suggested for IRF.$^{14}$ The presence at necropsy of severe atheromb atous plaques in arteries adjacent to areas of IRF hạs led to the hypothesis that insoluble lipid leaks into the perivascular tissue causing an inflammatory reactiof mediated by IgG. ${ }^{15}$ This so called "chronic periaortitis? also provides an explanation for the mediastin involvement of systemic fibrosis.

Ureteric obstruction has been reported in other immunologically-mediated vasculitic diseases. ${ }^{16}$ Ong

\section{.}


such case of Henoch-Schönlein purpura in an adult spontaneously resolved, as seen in our case of systemic Weber-Christian disease. Conservative management is not advocated in IRF.

The persistently low fourth component of complement is also not a well recognized feature of the disease. A recent review of Weber-Christian disease ${ }^{9}$ reported hypocomplementaemia $\left(C_{3} C_{4}\right)$ in a significant number of cases in addition to circulating $7 \mathrm{~S} \mathrm{IgM}$ at times of relapse. It is also of interest that hereditary deficiency of $\mathrm{C}_{4}$ is often associated with systemic lupus or a lupus-like syndrome, ${ }^{17}$ and systemic lupus is probably the most common identifiable cause of panniculitis. ${ }^{10}$ However, our patient did not have the clinical or laboratory features of systemic lupus erythematosus at any time during the course of his illness. We have been unable to date to examine other members of the family to determine whether the $\mathrm{C}_{4}$ deficiency might be hereditary in this case.

Another interesting aspect of our case was the progressive visual failure 10 years before presentation. Although ocular features have been described as complications of Weber-Christian disease, namely episcleral nodules, ${ }^{18}$ anterior and posterior uveitis ${ }^{19}$ and transient visual loss with macular haemorrhages, ${ }^{20}$ permanent blindness has not. The visual failure was preceded and accompanied by episodic fever and

\section{References}

1. Scully, R.E., Mark, E.J. \& McNeely, B.U. Case records of the Massachusetts General Hospital. N Engl J Med 1982, 306: 1035-1043.

2. Steinberg, B., Systemic nodular panniculitis. Am J Pathol 1953, 29: 1059-1081.

3. Milner, R.D.G. \& Mitchinson, M.J. Systemic WeberChristian disease. J Clin Pathol 1965, 18: 150-156.

4. Mitchinson, M.J. Systemic idiopathic fibrosis and systemic Weber-Christian disease. J Clin Pathol 1965, 18: 645-649.

5. Harbrecht, P.J. Variants of retroperitoneal fibrosis. Ann Surg 1967, 165: 388-401.

6. Castelman, B., Scully, R.E. \& McNeely, B.U. Case records of the Massachusetts General Hospital. N Engl J Med 1972, 287: 34-40.

7. Unger, H. Relapsing febrile nodular inflammation of the adipose tissue (Weber-Christian syndrome); report of a case with autopsy. $J$ Pathol 1946, 58: 175-185.

8. MacDonald, A. \& Feiwel, M. A review of the concept of Weber-Christian panniculitis with a report of five cases. Br J Dermatol 1968, 80: 355-361.

9. Panush, R.S., Yonker, R.A., Dlesk, A., Longley, S. \& Caldwell, J.R. Weber-Christian disease. Analysis of 15 cases and review of the literature. Medicine 1985, 64: $181-191$.

10. Diaz-Jouanen, E., De Horatius, R.J., Alarcon-Segovia, D. \& Messner, R.P. Systemic lupus erythematosus presenting as panniculitis (lupus profundus). Ann Intern Med 1975, 82: 376-379. elevation of the ESR and did not respond to corticosteroids. No clear diagnosis was reached at the time. Considering the subsequent evolution of his illness it is tempting to speculate that the visual failure was an early manifestation of Weber-Christian disease, and in view of the pathological changes on biopsy, that the vasculitis may have some relevance to its development. Freedman has also suggested that vasculitis may have been responsible for bilateral macular haemorrhages observed in a patient with Weber-Christian disease. ${ }^{20}$

The treatment of Weber-Christian disease has generally proved rather unsatisfactory. Our patient did not respond to corticosteroid therapy, an experience shared by others. ${ }^{8}$ We chose to use indomethacin because of a single uncontrolled but nevertheless convincing report of an excellent response to this agent. ${ }^{1}$ Our patient responded very satisfactorily in the short term but unfortunately the remission was not maintained. Hydroxychloroquine has been shown to be of some benefit ${ }^{9}$ but was subsequently unsuccessful in our case. Nevertheless, non-steroidal anti-inflammatory drugs and antimalarials should probably be tried before resorting to immunosuppressive therapy such as azathioprine ${ }^{21}$ and cyclophosphamide..$^{22,23}$

11. Coopersmith, N.H. \& Appelman, H.D. Multifocal fibrosclerosis with subcutaneous involvement. Am J Clin Pathol 1970, 55: 369-376.

12. Allen-Marsh, T.C. Weber-Christian panniculitis and autoimmune disease; a case report. J Clin Pathol 1976, 29: $144-149$.

13. Ciclitura, P.J., Wight, D.G.D. \& Dick, A.P. Systemic Weber-Christian disease, a case report with lipoprotein profile and immunological evaluation. $\mathrm{Br} J$ Dermatol 1980, 103: 685-692.

14. Mitchinson, M.J. Retroperitoneal fibrosis revisited. Arch Pathol Lab Med 1986, 110: 784-786.

15. Parum, S.D.V., Chadwick, D.R. \& Mitchinson, M.J. The localisation of immunoglobulin in periaortitis. Atherosclerosis 1986, 61: 117-123.

16. Powell, J.M., Ware, H. \& Williams, G. Recurrent ureteric obstruction in association with HenochSchönlein purpura. Postgrad Med J 1987, 63: 699-701.

17. Trappeiner, G., Hintner, H., Scholz, S., Albert, E., Linert, J. \& Wolff, K. Systemic lupus erythematosus in hereditary deficiency of the fourth component of complement. J Am Dermatol 1982, 7: 66-79.

18. Frayer, W.C. Ocular and adnexal changes associated with relapsing febrile non-suppurative panniculitis (Weber-Christian disease). Trans Am Ophthalmol Soc 1968, 66: 233-242.

19. Klein, B.A. Nodular non-suppurative panniculitis (Weber-Christian syndrome) with relapsing uveitis. Am J Ophthalmol 1959, 48: 230-234. 
20. Freedman, J. Ocular pathology associated with the Weber-Christian syndrome. Br J Ophthalmol 1972, 56: 896-898.

21. Hotta, T., Wakamatsu, Y., Matsamura, N. et al. Azathioprine-induced remission in Weber-Christian disease. South Med J 1981, 74: 234-236.

22. Martin, R.J., Michals, E.L. \& Voth, M.R. Cyclophosphamide-induced remission in WeberChristian disease; case report. Milit Med 1977, 142: 158-160.
23. Kirch, W., Duhrsen, U., Hoensch, H. \& Ohnhaus, E Cyclophosphamide-induced remission in WeberChristian disease. Rheumatol Int 1985, 5: 239-240. 